\title{
A binary hybrid replication strategy for improving availability and maintaining consistency of data in large scale mobile environments
}

\begin{abstract}
Data Management in mobile computing environments poses great challenges to the database researchers. One of the important challenges is to provide a data replication solution that maintains the consistency and improves the availability of replicated data. This paper addresses this problem for large scale distributed information systems that operate in mobile environments. Our solution represents a new binary hybrid replication strategy, in terms of its components and approach. The new strategy encompasses two components: replication architecture to provide a solid infrastructure for improving data availability and replication method to transfer data updates in a manner that achieves the consistency of data. The new strategy is a hybrid of both pessimistic and optimistic replication approaches, in order to exploit the features of each. The proposed strategy supports higher data availability and lower rate of inconsistencies as well as supports the mobility of users.
\end{abstract}

